\title{
Current status of transcatheter aortic valve replacement in India
}

\author{
Puneet Gupta $^{1}$, Sameer Arora ${ }^{2}$, Arman $_{\text {Qamar }}{ }^{3}$, Mohit Gupta $^{4}$, Ashok Seth $^{5}$ \\ ${ }^{1}$ Department of Cardiology, Janakpuri Super Speciality Hospital, New Delhi, India; ${ }^{2}$ Division of Cardiology, University of North Carolina, Chapel \\ Hill, NC, USA; ${ }^{3}$ TIMI Study Group, Division of Cardiology, Brigham and Women's Hospital, Harvard Medical School, Boston, MA, USA; \\ ${ }^{4}$ Department of Cardiology, Gobind Ballabh Pant Institute of Post Graduate Medical Education and Research, New Delhi, India; ${ }^{5}$ Fortis Escorts \\ Heart Institute, New Delhi, India \\ Contributions: (I) Conception and design: P Gupta, S Arora; (II) Administrative support: M Gupta, A Seth; (III) Provision of study materials or \\ patients: M Gupta, S Arora; (IV) Collection and assembly of data: P Gupta, A Qamar; (V) Data analysis and interpretation: P Gupta, M Gupta; (VI) \\ Manuscript writing: All authors; (VII) Final approval of manuscript: All authors. \\ Correspondence to: Puneet Gupta, MD, DM. Department of Cardiology, Janakpuri Super Speciality Hospital, Janakpuri, New Delhi 110058, India. \\ Email: pg.ucms@gmail.com.
}

\begin{abstract}
Transcatheter aortic valve replacement (TAVR) has proven to be the cornerstone therapy for patients with severe aortic stenosis (AS). India is currently overburdened with AS, both rheumatic and nonrheumatic. Although TAVR has revolutionized the treatment of non-rheumatic AS it does offer a ray of hope for rheumatic AS in future. It is difficult to measure the actual burden of AS in India due to lack of epidemiological studies in this population. Variable estimates have been given indicating a large number of patients eligible for TAVR. Despite its enormous potential, TAVR is still beyond the reach of an average Indian citizen. The progress for TAVR in India remains rather slow with obstacles at multiple levels. These include high costs, lack of coverage by insurance companies, continuous ignorance in government policies, lack of sufficient trained TAVR performing operators and lack of healthcare providers trained in post-TAVR care. Additionally, unique anatomical factors such as smaller body surface area, smaller annulus size, low coronary ostia take-off, smaller calibre of iliac and common femoral arteries pose unique challenges in terms of need for smaller hardware and increased chances of complications. It is crucial that the policy makers and healthcare personnel wake up to the benefits of this innovation and take appropriate steps to ease the dissemination of TAVR across the country.
\end{abstract}

Keywords: India; transcatheter aortic valve replacement (TAVR); MyVal

Submitted Apr 22, 2019. Accepted for publication May 27, 2019.

doi: $10.21037 / \mathrm{cdt} .2019 .05 .04$

View this article at: http://dx.doi.org/10.21037/cdt.2019.05.04

\section{Introduction}

Calcific aortic stenosis (AS) is an age-related degenerative disease with a high mortality if not treated $(1,2)$. The prognosis is worse than most other malignancies, with mortality being $50 \%$ at 2 years $(3,4)$. Data regarding epidemiology of valvular heart disease in India remains sparse likely due to lack of resources and maintenance of proper medical records. Therefore, the true prevalence of aortic valve disease in India is difficult to estimate and therefore, most of the prevalence estimates come from western literature (2). Although rheumatic heart disease contributes more than $60 \%$ of the valvular heart disease burden in India, trends have changed with rising life expectancies (5). Age-related degenerative disease has now emerged as the most common cause of isolated AS in India (5). Gradual increase has been observed in the number of deaths due to calcific aortic valve disease in South East Asia region, especially India, over the last decade (Figure 1) According to the global burden of disease study, in India, a large number of deaths are attributed to severe calcific nonrheumatic AS (6).

The efficacy of aortic valve replacement (AVR) in reducing mortality of AS patients has been proven without 


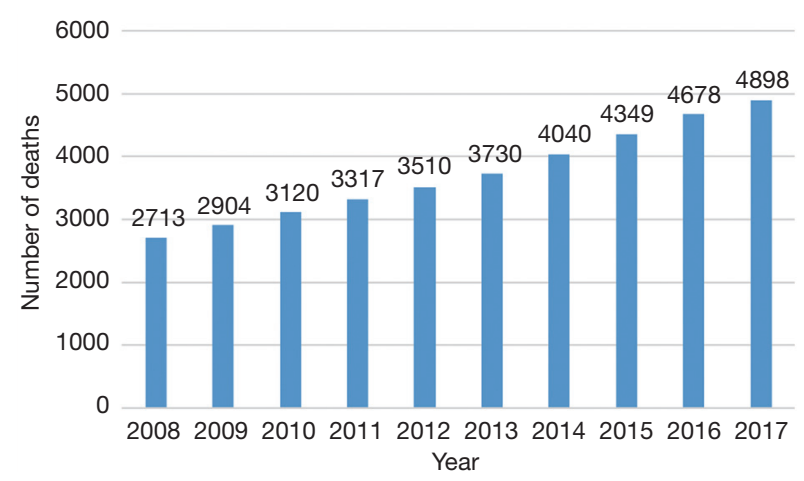

Figure 1 Increasing number of deaths in India due to calcific aortic stenosis.

doubt. In contrast, no medical therapy has been proven to lower mortality in these patients (4). Despite AVR being a class I recommendation for severe symptomatic AS according to American College of Cardiology/American Heart Association (ACC/AHA) guidelines (7), nearly one third patients are unable to undergo AVR because of comorbidities, frailty, left ventricular dysfunction, and advanced age (4). The management of such patients may dramatically change with the advent of this percutaneous therapy. According to a United Nations report, India had 10 million persons above the age of 80 years in 2013 (8). Based on recent Indian demographics profile data, 5-6\% of the total population of 1.25 billion (approximately 70 million people) are above the age of 65 years (9). Extrapolating western prevalence data to the Indian population, nearly 2.5-3 lakh patients with AS are likely to be eligible for transcatheter aortic valve replacement (TAVR). This is more than the combined estimates from Europe and North America.

The indications of TAVR have been rapidly expanding since its first introduction as a treatment for AS patients. Initially approved for inoperable and high-risk patients, it received U.S. Food and Drug Administration (FDA) approval for intermediate risk indication in 2017. Lowrisk trial results were reported this year and it seems that it would not be long before TAVR has an all-risk indication. The expansion of TAVR in India has been slow and steadily rising despite the first TAVR being performed in India in 2011. This is due to multiple factors and will be the focus of our review.

\section{Status of TAVR in India}

The first clinical experience with TAVR in India was in 2011 in 80-year-old female patient with severe AS who was denied surgery in view of high comorbidities. She underwent the first TAVR in India with a 26-mm Medtronic CoreValve through the transfemoral route and was discharged in a healthy condition. Two more patients underwent TAVR in February 2012 with the Medtronic CoreValve, out of which one of them required permanent pacemaker implantation (10). Currently TAVR is being done in around 30 centres across India out of which approximately seven centres are handling the majority of the TAVR load. This is far below the total number of cardiac catheterization laboratories available in India. The reason for this is probably the reluctance among the physicians and patients, regulatory body approval, lack of specialised training programs, need for dedicated heart team and specialised centres. Anatomic peculiarities of the Indian population-smaller aorta and peripheral vessel diameter, high prevalence of rheumatic heart disease, low coronary ostia, high prevalence of bicuspid aortic valve (BAV) and heavy calcification of aortic valve leaflets have major impact on the feasibility, prosthesis durability, procedural complications and long-term outcomes and need special attention.

\section{TAVR valves}

Two types of valves are currently approved by the U.S. FDA for TAVR: the CoreValve ${ }^{\circledR}$ Revalving system (Medtronic, Inc.; Minneapolis, Minnesota, USA) and the Edwards Sapien system (Edwards Lifesciences Corporation; Irvine, California, USA). Both valves have proved their effectiveness in the recent outcomes of the CoreValve US Pivotal and Partner trials, respectively. However, indigenous valve development is slowly picking up in India. TAVR procedures in India are done with both U.S. FDA approved and indigenous valves. Most common valves used are the CoreValve ${ }^{\circledR}$ Revalving system (Medtronic, Inc.; Minneapolis, Minnesota) and the Medtronic EvolutR valve (Figure 2). It is important to note that one indigenous valve-MyVal- has made a significant impact in the Indian market by virtue of its design, advanced delivery system and lower cost. Another self-expanding valve-Hydra aortic valve is slowly gaining popularity in Indian market.

\section{$M y \operatorname{Val}^{\mathrm{TM}}$}

It is an indigenously developed balloon-expandable TAVR system developed by Meril Life Sciences Pvt Ltd., India. This transcatheter heart valve (THV) system is Conformite 


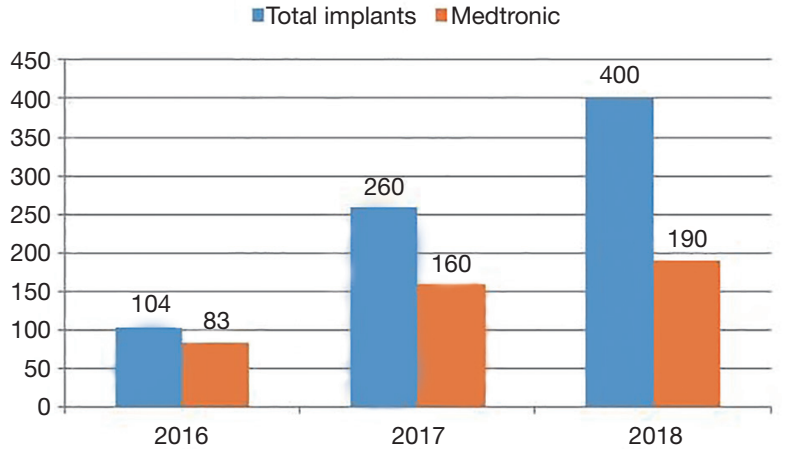

Figure 2 Total and Medtronic TAVR implants in India over the last 3 years. TAVR, transcatheter aortic valve replacement.

Européenne (CE) approved. The system includes a nickelcobalt alloy frame with increased radial strength. The precise orthotopic placement, positioning and deployment across the native annulus is facilitated by the unique honeycomb hybrid cell design structure with closed cells in the lower half for increased radial strength and open cells in the upper half for unjailing of the coronary ostia (Figure 3). The tri-leaflet structure of $\mathrm{MyVal}$ is made using the decellularized bovine pericardium tissue which has received anti-calcification treatment. External polyethylene terephthalate protective sealing cuff acts to reduce or nearly eliminate paravalvular leak (PVL). MyVal is available in a wide range of sizes depending on the diameter of the native annulus-20, 21.5, 23, 24.5, 26, 27.5, 29, or $32 \mathrm{~mm}$. The delivery of the THV system is done using a specially designed hi-flex, over-the-wire balloon catheter system (Navigator $^{\mathrm{TM}}$, Meril Life Sciences Pvt. Ltd., India). Direct crimping of MyVal THV over its Navigator delivery system eliminates un-necessary maneuvering of the device in descending aorta that is required in first generation balloon expandable THV. The Navigator delivery system with its ability to flex while crossing over the arch allows for minimal injury to arch anatomy. Dual nose cone on Navigator balloon ensures flawless crimping, precise positioning and predictable deployment of MyVal THV.

MyVal-1 study is a prospective multicentre first-inhuman study designed to evaluate the safety and efficacy of the MyVal THV system in 100 intermediate-tohigh risk patients of severe symptomatic AS. The study recruited 88 patients across 26 Indian sites from $30^{\text {th }}$ June 2017 to $17^{\text {th }}$ April 2019. Analysis of data collected from the first 30 patients who completed 12-month follow-up suggested favourable safety and efficacy of MyVal THV with no moderate/severe PVL, aortic regurgitation or need for new permanent pacemaker. Mean age of these 30 patients was $75.54 \pm 6.72$ years; $43.33 \%$ had coronary artery disease; mean Society of Thoracic Surgeons (STS) score was $6.42 \% \pm 1.78 \%$ and $100 \%$ patients were in New York Heart Association (NYHA) functional class II/III/ IV at pre-procedure. The mean aortic valve gradient, peak aortic-valve gradient, effective orifice area and trans-aortic velocity improved significantly over the 12-month followup period. In the subset of 30 patients, $96.55 \%$ and $100 \%$ were in NYHA functional class I/II at 30-day and 12-month post-implantation of MyVal THV, respectively. There was also significant improvement in the results of Kansas City Cardiomyopathy Questionnaire (KCCQ) score and the six-minute walk test from baseline to 12 -month followup (11). The analysis of the data after completion of the study will add further strength to the safety and efficacy of this THV system. The lower cost of this THV system as compared to the western devices has made it popular among the interventional cardiologists making the therapy available to more patients.

\section{Hydra aortic valve}

Hydra aortic bioprosthesis along with the Hydra aortic valve Delivery system was developed as a self-expanding aortic bioprosthesis system with a mechanism for recapturing of the prosthesis during deployment. It was developed by Vascular Innovations Co. Ltd., Nonthaburi, Thailand. After a preclinical study conducted in sheep which showed good overall biocompatibility, the first-inman study was conducted in 2014-2015. After a thorough heart team assessment, 15 patients of symptomatic severe AS underwent Hydra valve implant. All patients were symptomatic and two patients had severe symptoms (NYHA grade III). Procedural success was achieved in all patients. Five patients needed another valve due to suboptimal position of the first valve. More than mild para valvular leakage was seen in seven cases. One patient died due to rupture of aortic arch and one patient had a dissection of aorta which was managed conservatively. Two patients had a major vascular complication. Atrioventricular block requiring permanent pacemaker implantation was required in two patients. Post procedure valve gradients demonstrated no significant obstruction to flow (12). In conclusion, although the initial results appear promising, further studies with a larger sample size would be needed to prove the safety and efficacy of Hydra aortic valve. 


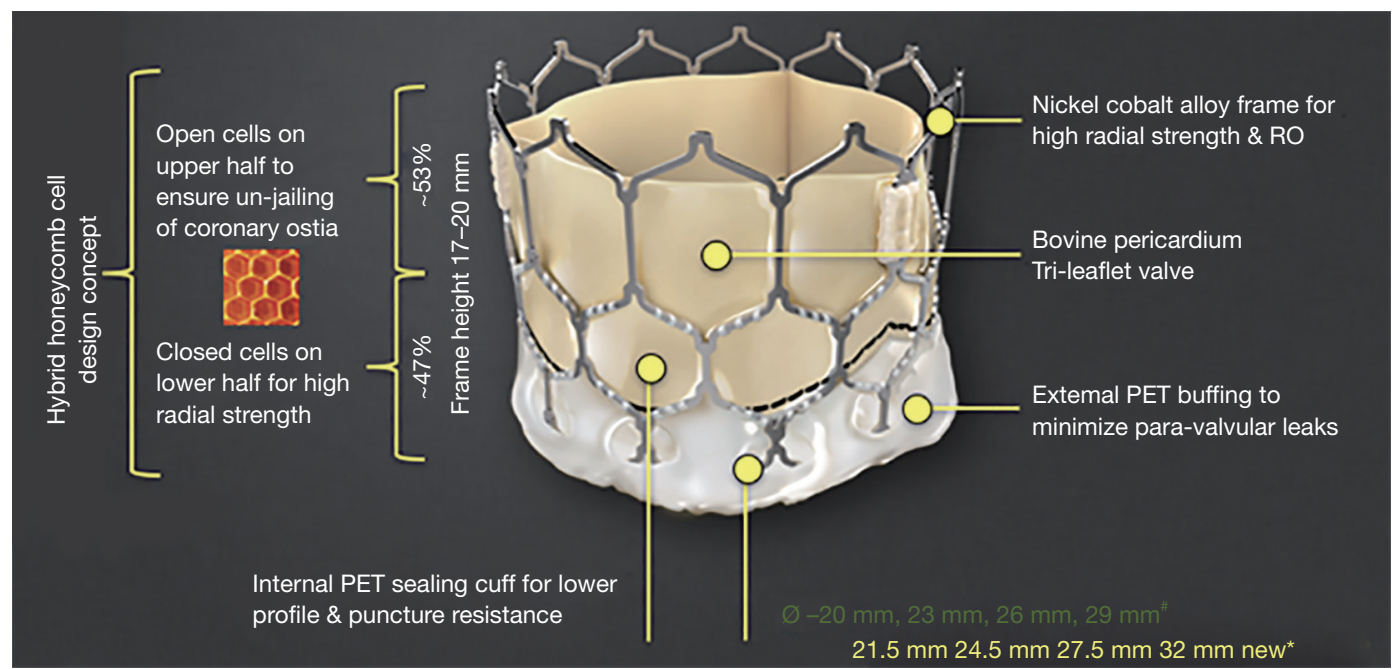

Figure 3 Unique hybrid geometry of MyVal. ", sizes available now; *, sizes available soon. PET, polyethylene terephthalate; RO, radio opacity.

\section{Unique challenges in Indian population}

\section{Cost and reimbursement policy}

Although not yet standardised, the estimated procedure cost for TAVR in India is approximately 35,000 U.S. dollars. Although the cost of TAVR in U.S. is almost twice that in India, it still remains a gigantic task for the Indian government to support the TAVR program in public health sector. In 2015, India spent only $3.9 \%$ of its gross domestic product on health sector, the lowest among the BRICS (Brazil, Russia, India, China, South Africa) countries (13). Even out of this $3.9 \%$, private health insurance accounts for only $5-10 \%$ of the expenditure, employers account for $9 \%$ while personal expenditure accounts for an astounding $82 \%$ (14). Hence, it remains a challenge for an average Indian to be able to support a TAVR procedure from his pocket. The cost of surgical AVR is much less than that of TAVR and therefore, remains the preferred option. Technical advancements, advent of indigenously developed valves, availability of locally manufactured devices, increased patient volumes, dedicated physician training programs and procedure simplification may aid in increasing the TAVR uptake and reducing the cost of the procedure in India.

\section{Regulatory body approval}

Medical devices and In Vitro Diagnostic Devices are regulated by the Drug Controller General of India (DCGI) within the Central Drugs Standard Control Organization
(CDSCO), part of the Ministry of Health and Family Welfare. The regulatory framework for medical devices is based on the Medical Device Rules, 2017. Indigenously developed THV system, MyVal recently got the approval from CDSCO and became the first Indian company in the world to enter the TAVR market which is currently dominated by Medtronic and Edwards Life Sciences. Since the procedure is gaining popularity in India and after the approval of this indigenous valve, it may be expected that TAVR will gain widespread acceptance throughout the country and will be available at lower costs. Similar efforts from the industry and support from the government will encourage development of similar newer devices.

\section{Acquiring proficiency in TAVR}

The American Association for Thoracic Surgery (AATS), American College of Cardiology Foundation (ACCF), Society for Cardiovascular Angiography and Interventions (SCAI), and STS have jointly identified operator and institutional requirements for performing TAVR (15). Operators must have a comprehensive knowledge of aortic valve disease, its epidemiology and local patterns, invasive and non-invasive diagnostics, natural history, medical and surgical treatment and its outcomes. Sufficient skill in echocardiography and interpretation of computed tomography (CT) films of the iliofemoral vessels and aortic valvular anatomy is also required. TAVR has a steep learning curve and many interventionists in India are still at 
the lower part of the curve.

\section{Anatomical factors}

In general, Asian individuals have a smaller body surface area (BSA), smaller annulus size and low coronary ostia take-off (16-18). The mean diameter of the iliac and the common femoral artery is also smaller in Asians by about $1 \mathrm{~mm}$ (19). These differences raise concerns for increased chances of complications and points towards need for smaller devices and better delivery systems. Another anatomic peculiarity among the Indian patients is the high prevalence of BAV. In an echocardiographic study conducted at a tertiary care centre which analysed 1,30,000 echocardiograms, isolated AS was found in $7.3 \%$ cases and BAV accounted for $33.9 \%$ of these cases (5). Presence of $\mathrm{BAV}$ has been associated with greater aortic angulation, leaflet calcium burden and ascending aortic dimensions as compared to tricuspid valves (20) which may have an impact on the procedural success and outcome. Moreover, the dimensions of the sinus of Valsalva and of the sinotubular junction in the Asian population are smaller and the coronary ostia lower than those of Western patients, potentially increasing the risk of coronary occlusion during TAVR (17).

\section{Future directions}

\section{Strategies for a successful TAVR program in India}

Establishment of nationwide training and education programs is needed to widen the scope of TAVR in India. Physicians, cardiologists and cardiac surgeons who are likely to encounter patients of AS must be made aware of the indications, the feasibility and ease of the procedure in trained hands, the results and outcomes of the trials conducted so far.

The regulatory bodies must wake up to the fact that TAVR is the future of aortic valve disease treatment and ease the procedure of new valve approval and introduce TAVR program in the public health sector.

The device and pharmaceutical industry also should plan and devise strategies in collaboration with the government to propagate TAVR in the Indian subcontinent considering the immense need and limited resources. They can also contribute in training of physicians, bringing about awareness among the public and physicians, research and development of newer valves suitable for the unique Indian population.

Collaboration among the TAVR enthusiasts such as interventional cardiologists, cardiothoracic surgeons, echocardiographers, cardiac anaesthetists, hospital paramedics and the industry should be promoted at all levels to encourage adoption and training of a new interventional skill that would benefit patients. Continuous research and development are needed to bring an affordable TAVR program to a large population.

\section{Conclusions}

Since its introduction, the technology of TAVR has undergone rapid expansion and seems to be on its way to an all-risk indication. However, its dissemination in India has been hindered by bottlenecks such as cost, poor reimbursement, lack of indigenous valves and hardware, lack of structured training, poor heart team concept and peculiar anatomical features of Indian population. With increasingly ageing population, TAVR will likely play a critical role in the future of cardiovascular health of India. TAVR has now consolidated its role as a critical option for aortic valve disease and it is time that regulatory authorities take cognizance of the huge unmet need of this percutaneous therapy and take steps towards wide implementation of TAVR.

\section{Acknowledgments}

None.

\section{Footnote}

Conflicts of Interest: The authors have no conflicts of interest to declare.

\section{References}

1. Stewart BF, Siscovick D, Lind BK, et al. Clinical factors associated with calcific aortic valve disease. Cardiovascular Health Study. J Am Coll Cardiol 1997;29:630-4.

2. Osnabrugge RL, Mylotte D, Head SJ, et al. Aortic stenosis in the elderly: disease prevalence and number of candidates for transcatheter aortic valve replacement: a meta-analysis and modeling study. J Am Coll Cardiol 2013;62:1002-12.

3. Ross J Jr, Braunwald E. Aortic stenosis. Circulation 1968;38:61-7.

4. Varadarajan P, Kapoor N, Bansal RC, et al. Survival in 
elderly patients with severe aortic stenosis is dramatically improved by aortic valve replacement: Results from a cohort of 277 patients aged $>$ or $=80$ years. Eur J Cardiothorac Surg 2006;30:722-7.

5. Manjunath CN, Srinivas $\mathrm{P}$, Ravindranath KS, et al. Incidence and patterns of valvular heart disease in a tertiary care high-volume cardiac center: a single center experience. Indian Heart J 2014;66:320-6.

6. GBD Results Tool I GHDx. Available online: http://ghdx. healthdata.org/gbd-results-tool

7. Nishimura RA, Otto CM, Bonow RO, et al. 2017 AHA/ ACC Focused Update of the 2014 AHA/ACC Guideline for the Management of Patients With Valvular Heart Disease: A Report of the American College of Cardiology/ American Heart Association Task Force on Clinical Practice Guidelines. Circulation 2017;135:e1159-e1195.

8. World Population Ageing 2013. Available online: http://www.un.org/en/development/desa/population/ publications/pdf/ageing/WorldPopulationAgeing2013.pdf

9. Census of India Website: SRS Statistical Report 2013 [Internet]. [cited 2019 Jan 6]. Available online: http://www. censusindia.gov.in/vital_statistics/SRS_Reports_2013.html

10. Seth A, Rastogi V, Kumar V, et al. Transcatheter aortic valve implantation with Core Valve: first Indian experience of three high surgical risk patients with severe aortic stenosis. Indian Heart J 2013;65:395-9.

11. Seth A. MyVal-1 Study: One-year clinical outcomes of India's first indigenously designed and manufactured TAVR system. Presented as a Late Breaking Trial at the EuroPCR 2019; May 22nd, 2019; Paris, France.

12. Srimahachota $S$, Buddhari $W$, Songmuang SB, et al. Firstin-man study of transcatheter aortic valve implantations in aortic stenosis using the Hydra self-expanding bioprosthesis. AsiaIntervention 2017;3:177-82.

13. GHO । By category | Current health expenditure (CHE)

Cite this article as: Gupta P, Arora S, Qamar A, Gupta M, Seth A. Current status of transcatheter aortic valve replacement in India. Cardiovasc Diagn Ther 2020;10(1):83-88. doi: 10.21037/cdt.2019.05.04 as percentage of gross domestic product (GDP) (\%). Data by country. WHO. Available online: http://apps.who.int/ gho/data/view.main.GHEDCHEGDPSHA2011v?lang=en

14. Media C. Health Insurance and Telecom Markets - A Comparative Study [Internet]. Centre for Public Policy Research (CPPR). 2015. Available online: https://www. cppr.in/article/health-insurance-and-telecom-markets-acomparative-study/

15. Tommaso CL, Bolman RM 3rd, Feldman T, et al. Multisociety (AATS, ACCF, SCAI, and STS) expert consensus statement: operator and institutional requirements for transcatheter valve repair and replacement, Part 1: transcatheter aortic valve replacement. Ann Thorac Surg 2012;93:2093-110.

16. Yoon SH, Ahn JM, Hayashida K, et al. Clinical Outcomes Following Transcatheter Aortic Valve Replacement in Asian Population. JACC Cardiovasc Interv 2016;9:926-33.

17. Watanabe Y, Hayashida K, Takayama M, et al. First direct comparison of clinical outcomes between European and Asian cohorts in transcatheter aortic valve implantation: the Massy study group vs. the PREVAIL JAPAN trial. J Cardiol 2015;65:112-6.

18. Inohara T, Hayashida K, Watanabe Y, et al. Streamlining the learning process for TAVI: Insight from a comparative analysis of the OCEAN-TAVI and the massy registries. Catheter Cardiovasc Interv 2016;87:963-70.

19. Chiam PT, Koh AS, Ewe SH, et al. Iliofemoral anatomy among Asians: implications for transcatheter aortic valve implantation. Int J Cardiol 2013;167:1373-9.

20. Jilaihawi H, Wu Y, Yang Y, et al. Morphological characteristics of severe aortic stenosis in China: imaging corelab observations from the first Chinese transcatheter aortic valve trial. Catheter Cardiovasc Interv 2015;85 Suppl 1:752-61. 